\title{
Relationship between single nucleotide polymorphisms in the 3'-untranslated region of the vascular endothelial growth factor gene and susceptibility to diabetic peripheral neuropathy in China
}

\author{
Xinhuan Zhang ${ }^{1,2}$, Zhongan Sun ${ }^{3}$, Hui Jiang ${ }^{1}$, Xiao Song
}

\author{
1Department of Endocrinology, Affiliated Hospital of Taishan Medical University, Tai' an, \\ Shandong, China \\ 2Department of Endocrinology, Shandong Provincial Hospital Affiliated to Shandong \\ University, Jinan, China \\ ${ }^{3}$ Department of Endocrinology, No. 88 Hospital, Tai'an, Shandong, China
}

Submitted: 7 December 2012

Accepted: 15 May 2013

Arch Med Sci 2014; 10, 5: 1028-1034

DOI: 10.5114 /aoms.2013.39381

Copyright (c) 2014 Termedia \& Banach

\begin{abstract}
Introduction: This study is to elucidate the relationship between a 936C/T mutation at the 3'-untranslated region of the human vascular endothelial growth factor (VEGF) gene and diabetic peripheral neuropathy (DPN).

Material and methods: All subjects recruited in this study were divided into DM (diabetes without neuropathy, retinopathy or nephropathy), DPN (diabetes with peripheral neuropathy only) and healthy control groups. The gene polymorphism was determined by polymerase chain reaction-restriction fragment length polymorphism, as well as other clinical methods including serum VEGF by ELISA.

Results: The C allele frequency and CC genotype frequency in the DPN group were higher than those in the NC group and DM group. The T allele frequency and CT+TT genotype (carrying the T allele) frequency in the DPN group were lower than those in the NC group $\left(\chi^{2}=19.051\right.$ and 18.533, both $\left.p<0.001\right)$ and DM group $\left(\chi^{2}=11.117\right.$ and 11.156 , both $\left.p=0.001\right)$. However, there was no statistically significant difference in the three genotype $(C C / C T+T T)$ frequencies and allele $(C / T)$ frequencies between the DM group and the NC group. The multivariate logistic regression analysis showed that the levels of glycated hemoglobin $\left(\mathrm{HbA}_{1 \mathrm{c}}\right)$, total cholesterol (TC), low density lipoprotein cholesterol (LDL-C) and plasma VEGF positively correlated with DPN, while the $936 \mathrm{C} / \mathrm{T}$ gene polymorphism of VEGF negatively correlated with DPN.
\end{abstract}

Conclusions: Allele $936 \mathrm{C}$ of VEGF may serve as a genetic marker susceptible to DPN, while allele 936T may be a protective genetic marker of DPN.

Key words: vascular endothelial growth factor, polymorphisms, type 2 diabetes mellitus, diabetic peripheral neuropathy.

\section{Introduction}

Vascular endothelial growth factor (VEGF), isolated by Ferrara and DavisSmyth [1] in 1989, is a potent endothelial cell mitogen that promotes the proliferation of vascular endothelial cells. Vascular endothelial growth factor plays a pivotal role in the regulation of physiological and pathological angiogenesis. There have been lots of reports on the role of VEGF-mediated angiogenesis and vascular permeability changes in chronic microvas-

\author{
Correspondence author: \\ Xinhuan Zhang \\ Department of Endocrinology \\ Affiliated Hospital \\ of Taishan Medical University \\ Tai'an, 271000 \\ Shandong, China \\ Phone: 865386131935 \\ Fax: 865316209988 \\ E-mail: kathy0531@126.com
}


cular complications of diabetes. The association of VEGF and diabetic neuropathy has attracted attention in recent years. For example, Deguchi et al. [2] found that the serum VEGF level was significantly increased in diabetic neuropathy. Currently it is believed that VEGF affects nerve fibers by changing the structure and function of endothelial cells. Vascular endothelial growth factor can increase capillary permeability, promote the synthesis of extracellular matrix and promote the proliferation and hypertrophy of endothelial cells. Also VEGF can induce basement membrane thickening and aggravate capillary obstruction. In addition, VEGF can impair the barrier function of the basement membrane and increase the endoneurial vascular permeability, leading to nerve fiber ischemia and hypoxia, and thus contributing to the occurrence of neuropathy [3]. On the other hand, Sondell et al. [4] and Tremolada et al. [5] reported that where there was ischemia and hypoxia, high expression of VEGF was protective for ischemic neuronal damage. This was because VEGF could not only promote angiogenesis of the nervous system but also have direct neurotrophic effects on the nervous system.

There are multiple polymorphism loci in the VEGF gene. The 936C/T gene polymorphism located in the $3^{\prime}$-untranslated region (3'-UTR) is associated with the expression level of VEGF [6, 7]. However, there have been no reports on the association of VEGF 936C/T polymorphism and type 2 diabetes with peripheral neuropathy so far. In this study we examined the relationship between VEGF 936C/T polymorphism and VEGF expression level in type 2 diabetic peripheral neuropathy.

\section{Material and methods}

\section{Subjects}

According to the diagnostic and exclusion criteria and typing standard for diabetes suggested by the WHO experts in 1999, unrelated patients with type 2 diabetes continuously attending the same hospital during 3 years were enrolled in this study. Diabetic peripheral neuropathy was diagnosed based on the diagnostic criteria suggested by Practical Endocrinology [8]. The clinical symptoms (such as symptoms of motor and/or sensory neuropathy) were examined and an electromyography (EMG) examination (for conduction disorders of motor and/or sensory nerves) was performed. A total of 388 patients with type 2 diabetes were selected. The pure diabetes group (DM) was composed of 184 cases, of which 92 cases were male and 92 cases were female, with a median age of $62(36-87)$ years, free blood glucose level of $9.61 \pm 1.02 \mathrm{mmol} / \mathrm{l}$ and disease duration of $9.28 \pm 4.56$ years. The diabetic peripheral neuropathy group (DPN) was composed of 204 cases, of which 104 cases were male and 100 cases were female, with a median age of 59 (38-85) years, free blood glucose level of 9.22 $\pm 3.56 \mathrm{mmol} / \mathrm{l}$ and diabetes duration of $9.98 \pm 4.34$ years. Two hundred and forty healthy individuals (male 124, female 116), with a median age of 60 (4178) years and free blood glucose level of $4.97 \pm 0.63$ $\mathrm{mmol} / \mathrm{l}$ were enrolled as the healthy control group (NC). They all underwent physical examination at the same hospital during the same period. All the 240 healthy individuals were assessed as normal in the oral $75 \mathrm{~g}$ glucose tolerance test (OGTT test). All of the subjects were Han people from Shandong Province, unrelated to one another. Subjects with nerve, retina, chronic cardiopulmonary diseases, liver and other organ diseases were excluded. The study was performed in accordance with the Declaration of Helsinki and was approved by the ethics committee of the hospital. Written informed consent was obtained from all subjects in advance.

\section{Clinical and biochemical features}

The following clinical and biochemical data of each subject were collected. Body mass index (BMI = body weight $(\mathrm{kg}) /$ height $(\mathrm{m})^{2}$ ) was measured. Fasting venous blood was taken, partly for the blood biochemistry analysis and VEGF detection, and partly for extraction of DNA. Fasting blood glucose (FBG) was measured by glucose oxidase. Blood lipids, liver function, renal function and electrolyte were analyzed by an automatic biochemical analyzer. The low pressure liquid chromatography column method was used to measure glycated hemoglobin (glycohemoglobin $\mathrm{HbA}_{1 \mathrm{c}}$ ).

\section{ELISA}

The serum VEGF level was detected by a ELISA kit purchased from Wuhan Boster Biological Engineering Co., Ltd. In this study, the double antibody sandwich ELISA (DAS-ELISA) method was used. Experiments were performed strictly in accordance with the instructions. Briefly, $2 \mathrm{ml}$ of fasting venous blood were taken. Sera were separated within 30 min by cryogenic centrifugation and stored at $-70^{\circ} \mathrm{C}$ in a refrigerator. The anti-human VEGF monoclonal antibody was coated on the microtiter plate. Then standard samples and test samples with VEGF were added to bind the monoclonal antibody. After incubation, biotinylated anti-human VEGF was added and immune complexes were formed on the plate. Horseradish peroxidase-labeled streptavidin was added to bind the biotin on the antibody. After adding substrate and finally sulfuric acid to terminate the reaction, the OD value at $450 \mathrm{~nm}$ was measured. Vascular epidermal growth factor concentration is proportional to the OD $450 \mathrm{~nm}$ value. Vascular endothelial growth factor concentration in the samples was obtained by the standard curve. 


\section{EMG measurement}

The diagnostic criteria [9] for diabetic peripheral neuropathy included clinical symptoms, clinical signs, the electrophysiological diagnosis, quantified sensory evaluation and the evaluation of autonomic function. Diabetes patients with at least one aspect of abnormalities were diagnosed with diabetic peripheral neuropathy. The motor nerve conduction velocity (MCV) and the sensory nerve conduction velocity (SCV) of each patient were measured by Keypoint 11 surface electrode EMG (Denmark). To obtain the MCV value, stimuli were added at both the distal end and the proximal end. Signals were received at the corresponding distal end of the muscles. The $M C V$ value was the quotient of the distance between the two stimulation points divided by the latency difference between the two points. The SCV value was obtained by the inverse method. Feeling waveform was made automatically by the average stacking technique of the machine. The SCV value was the quotient of the distance between the two stimulation points divided by the latency. The standard nerve conduction velocity was referred to Tang [10].

\section{Polymerase chain reaction-restriction fragment length polymorphism}

Genomic DNA was extracted using a DNA extraction kit (TaKaRa). For polymerase chain reaction (PCR) amplification, peripheral blood leukocyte genomic DNA was used as a template. The primer sequences were designed according to the cDNA sequence of the VEGF206 isoform (GenBank accession numbers AF024710 and S85192) and Renner [6] and synthesized by Songon Biotech (Shanghai)
Co., Ltd. The sequences for the upstream and downstream primers were $5^{\prime}$-AAGGAAGAGGAGACTCTGCGCAGAGC-3', 5'-TAAATGTATGTATGTGGGTGGG TGTGTCTACAGG-3'. Restriction enzyme analysis: $10 \mu \mathrm{l}$ of PCR products were digested with $5 \mathrm{U}$ of restriction endonuclease Nla III (New England Biolabs). Digestion products and the reference marker were analyzed on 4\% low-melting agarose gel. After electrophoresis, the gel was photographed and analyzed.

\section{Statistical analysis}

All data were processed by SPSS 10.0 software. The Hardy-Weinberg equilibrium test was used for the examination of the gene frequencies of each group. Normally distributed data were expressed as mean \pm standard deviation $(\bar{x} \pm s)$. One-way analysis of variance (ANOVA) was used for the comparison between the clinical and biochemical data of each group. Distribution of genotypes and alleles was compared by $\chi^{2}$ test or $\mathrm{R} \times \mathrm{C}$ table column associated analysis. Logistic regression analysis was performed to analyze the related risk factors for type 2 diabetes neuropathy.

\section{Results}

There are significant decreases in the frequency of 936T allele and VEGF 936 (CT+TT) genotype in patients with diabetic neuropathy.

Genotypes were analyzed by polymerase chain reaction-restriction fragment length polymorphism (PCR-RFLP). The sketch map for human 3'UTR of VEGF is shown in Figure $1 \mathrm{~A}$ : the location of the upstream primer is from number 148 to 173 and the

A

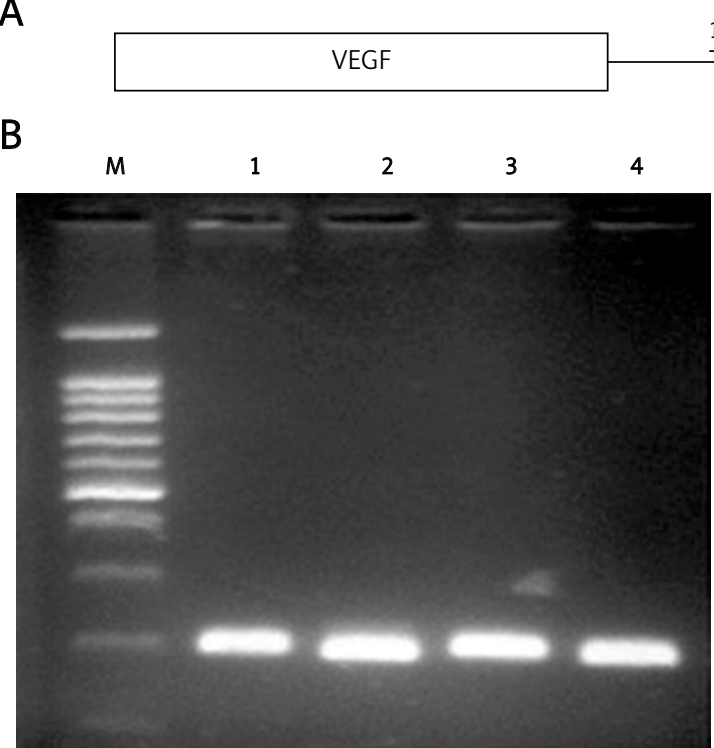

C

$\begin{array}{lllll}\text { CT } & \pi & C C & M\end{array}$

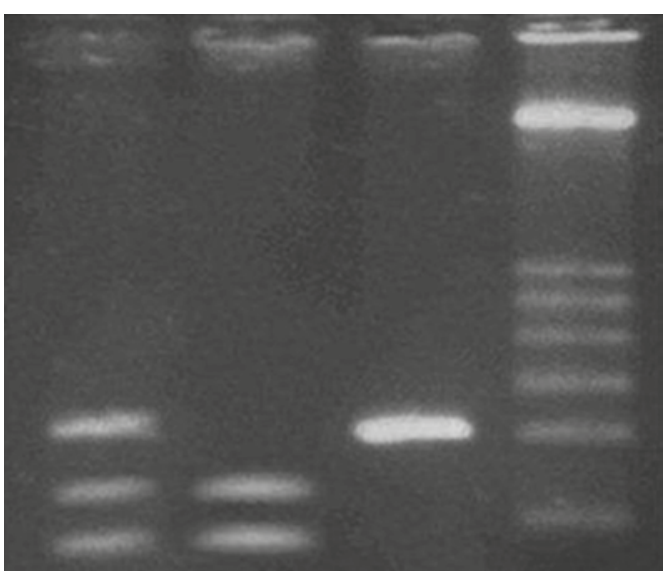

Figure 1. SNP polymorphisms at C936T locus of the VEGF gene. A - The C936T locus in the 3' UTR of the VEGF gene. B-PCR products and 100 bp ladder marker. Lane 1: 100 bp ladder marker; lane 2-5: PCR products. C- Genotype of VEGF gene C936T variant. lane 1: CT genotype; lane 2: TT genotype; lane 3: CC genotype; lane 4; 100 bp ladder marker 
Table I. Distribution of VEGF genotype and allele frequencies of the subjects in the NC, DM, and DPN group

\begin{tabular}{|lccccc|}
\hline Group & Cases $(n)$ & \multicolumn{2}{c|}{ Genotype frequency, $n(\%)$} & \multicolumn{2}{c|}{ Allele frequency, $n$ (\%) } \\
\cline { 2 - 6 } & & CC & CT+TT & C & T \\
\hline NC group & 240 & $141(58.75)$ & $99(41.25)$ & $365(76.25)$ & $115(23.75)$ \\
\hline DM group & 184 & $115(62.50)$ & $69(37.50)$ & $289(78.53)$ & $79(21.47)$ \\
\hline DPN group & 204 & $159(77.94)^{a, b}$ & $45(22.06)^{a}, \mathrm{~b}$ & $357(87.50)^{a}, \mathrm{~b}$ & $51(12.50) \mathrm{a}, \mathrm{b}$ \\
\hline
\end{tabular}

Compared with the NC group, $a_{p}<0.05$; compared with the DM group, $b_{p}<0.05$

downstream primer is from number 322 to 355. The 936C/T single-nucleotide polymorphisms in the VEGF gene lies in codon 232 (according to the nucleotide sequence from GenBank accession numbers AF024710). Figure $1 \mathrm{~B}$ shows the representative results of PCR amplification. The length of the PCR products was $208 \mathrm{bp}$. Then the PCR products were digested with Nla III and the electrophoresis results are shown in Figure $1 \mathrm{C}$. The digestion products were for CC genotype a 208 bp fragment, for TT genotype $122 \mathrm{bp}$ and $86 \mathrm{bp}$ fragments, and for CT genotype three fragments of $208 \mathrm{bp}, 122 \mathrm{bp}$ and $86 \mathrm{bp}$.

Polymerase chain reaction-restriction fragment length polymorphism test results showed that the $936 \mathrm{C} / \mathrm{T}$ polymorphism is prevalent in patients with type 2 diabetes and healthy control subjects (Table I). As shown in Table I, the genotype distribution in the NC group, DM group and DPN group did not show a significant deviation from the HardyWeinberg equilibrium $\left(\chi^{2}\right.$ value of $0.620,0.440$ and 3.240 , respectively, $p=0.733, p=0.802, p=0.198$, respectively). Due to the extremely low frequency of TT genotype, carriers of the T allele were combined into CT+TT genotype before analysis according to the statistical method suggested by Krippl et al. [7]. The T allele frequency and CT+TT genotype (carrying the T allele) frequency in the DPN group were significantly lower than those in the NC group $\left(\chi^{2}=19.051\right.$ and 18.533, $p<0.001$ and $p<0.001$, respectively) and DM group $\left(\chi^{2}=11.117\right.$ and 11.156, $p=0.001$ and 0.001 , respectively). However, there was no statistically significant difference $\left(\chi^{2}=0.240\right.$ and $0.258, p=0.624$ and 0.611 , respectively) in the three genotype $(\mathrm{CC} / \mathrm{CT}+\mathrm{TT})$ frequencies and allele $(\mathrm{C} / \mathrm{T})$ frequencies between the DM group and the NC group.

\section{The polymorphism $936 \mathrm{C} / \mathrm{T}$ of VEGF is associated with the levels of plasma VEGF, $\mathrm{HbA}_{1 c}$, blood glucose, blood lipids, and C-peptide}

As shown in Table II, in all subjects, the clinical characteristics in different VEGF polymorphism genotypes such as sex ratio, age, duration of disease, blood pressure, BMI and high-density lipoprotein cholesterol (HDL-C) showed no significant difference $(p>0.05)$ among the three different genotypes CC, CT and TT. Compared with CT+TT genotype (carrying the $T$ allele), the blood glucose, blood lipids, $\mathrm{HbA}_{1 \mathrm{c}}$ and VEGF expression levels of the CC genotype were higher, while the $C$-peptide levels of the CC genotype were significantly lower $(p<0.01, p<0.05)$. There were no significant differences in sex ratio, age, blood pressure and $\mathrm{BMI}$ among the NC group, DM group and DPN group $(p>0.05)$. Also there were no significant differences in $\mathrm{HbA}_{1 c}$, blood glucose and blood lipid levels between the DM group and DPN group $(p>0.05)$. However, the $\mathrm{HbA}_{1 c}$, blood glucose and blood lipid levels in the DM group and DPN group were higher than those in the NC group $(p<0.05)$.

\section{The incidence of diabetic peripheral neuropathy is significantly lower in the VEGF $936(\mathrm{CT}+\mathrm{TT})$ genotype (carrying the $T$ allele) group}

As shown in Table III, in diabetic patients, the incidence of diabetic peripheral neuropathy in the CC genotype group was $57.97 \%$, higher than the CT+TT genotype group $\left(39.29 \%, \chi^{2}=5.578\right.$,

Table II. Comparison of clinical characteristics among subjects in different VEGF polymorphism genotypes $(\bar{x} \pm s)$

\begin{tabular}{|c|c|c|c|}
\hline Parameter & $\pi$ & $\mathrm{CT}$ & $\mathrm{CC}$ \\
\hline$N(\mathrm{M} / \mathrm{F})$ & $32(18 / 14)$ & $181(90 / 91)$ & $415(210 / 205)$ \\
\hline Age [years] & $59(36-83)$ & $58(39-83)$ & $60(41-87)$ \\
\hline Duration [years] & $9.04 \pm 3.62$ & $9.06 \pm 3.61$ & $9.04 \pm 3.85$ \\
\hline FBG [mmol/l] & $7.38 \pm 1.73$ & $9.06 \pm 2.61^{a}$ & $9.32 \pm 1.86^{b, c}$ \\
\hline PBG [mmol/l] & $12.26 \pm 2.65$ & $13.06 \pm 3.17 a$ & $13.21 \pm 4.08^{b, d}$ \\
\hline $\mathrm{HbA}_{1 \mathrm{c}}[\%]$ & $6.98 \pm 1.78$ & $7.99 \pm 1.32^{\mathrm{a}}$ & $7.96 \pm 2.1^{\mathrm{a}}$ \\
\hline $\mathrm{BMI}\left[\mathrm{kg} / \mathrm{m}^{2}\right]$ & $22.16 \pm 2.54$ & $22.85 \pm 2.43$ & $22.79 \pm 2.56$ \\
\hline $\mathrm{TC}[\mathrm{mmol} / \mathrm{l}]$ & $5.42 \pm 1.34$ & $5.56 \pm 1.54^{a}$ & $5.87 \pm 1.46^{b, c}$ \\
\hline $\mathrm{TG}[\mathrm{mmol} / \mathrm{l}]$ & $1.51 \pm 0.65$ & $1.65 \pm 0.59^{a}$ & $1.75 \pm 0.87 a, b$ \\
\hline $\mathrm{HDL}-\mathrm{C}[\mathrm{mmol} / \mathrm{l}]$ & $1.68 \pm 0.31$ & $1.71 \pm 0.25$ & $1.73 \pm 0.43$ \\
\hline LDL-C [mmol/ll] & $2.71 \pm 0.46$ & $2.81 \pm 0.36^{a}$ & $2.84 \pm 0.39 \mathrm{~b}, \mathrm{~d}$ \\
\hline $\mathrm{SBP}[\mathrm{mm} \mathrm{Hg}]$ & $132.43 \pm 12.34$ & $126.65 \pm 11.34$ & $126.65 \pm 11.87$ \\
\hline $\mathrm{DBP}[\mathrm{mm} \mathrm{Hg}]$ & $81.65 \pm 8.34$ & $79.65 \pm 8.51$ & $81.45 \pm 7.99$ \\
\hline VEGF [ng/l] & $445.32 \pm 119.76$ & $\begin{array}{c}598.38 \\
\pm 122.44 a\end{array}$ & $\begin{array}{c}621.33 \\
\pm 110.23 \mathrm{~b}, \mathrm{c}\end{array}$ \\
\hline
\end{tabular}

Compared to TT genotype, $a_{p}<0.05, b_{p}<0.01$; compared to CT genotype, $c_{p}<0.05, d_{p}<0.01$ 
Table III. Distribution of different VEGF polymorphism genotypes in type 2 diabetes with or without DPN

\begin{tabular}{|lccc|}
\hline & DPN, $n(\%)$ & DM, $n(\%)$ & Total \\
\hline CC & $159(57.97)$ & $115(42.02)$ & 274 \\
\hline CT & $39(39.58)^{\mathrm{a}}$ & $59(60.42)^{\mathrm{a}}$ & 98 \\
\hline $\mathrm{CT}+\mathrm{TT}$ & $45(39.29)^{\mathrm{b}, \mathrm{c}}$ & $69(60.71)^{\mathrm{b}, \mathrm{c}}$ & 114 \\
\hline
\end{tabular}

${ }^{a}$ Compared to CC genotype $\left(\chi^{2}=4.837, O R=2.105, p=0.002\right) ;{ }^{b}$ com pared to CC genotype $\left(\chi^{2}=5.578, O R=2.132, p=0.001\right)$; ${ }^{C}$ compared to CT genotype $\left(\chi^{2}=0.001, O R=1.014, p=0.962\right)$

$p=0.001)$ and CT genotype group (39.58\%, $\chi^{2}=4.837$, $p=0.002)$. Compared with carriers of CT+TT genotypes and $\mathrm{CT}$ genotypes, the relative neuropathy occurrence risk (OR) for carriers of the CC genotype was 2.132 and 2.105, respectively, while the incidence of diabetic peripheral neuropathy was not significantly different between the CT genotype group and the CT+TT genotype group $\left(\chi^{2}=0.001\right.$, $p=0.962)$.

The $936 \mathrm{C}$ to T mutation in the 3'-untranslated region of VEGF may be a protective factor against DPN

Logistic regression analysis was performed to assess the independent role of the VEGF genotype and other variables (including $\mathrm{HbA}_{1 c}$ level, $\mathrm{TC}$ level, LDL-C level, VEGF concentration and VEGF 936C $\rightarrow$ T) in the incidence of DPN. In this analysis, the presence or absence of DPN was used as the dependent variable. As shown in Table IV, the 936C/T polymorphism in the 3'-UTR of the VEGF gene was negatively related to the occurrence of DPN $(\beta=-1.132$, OR = $0.461, p=0.004,95 \% \mathrm{Cl}: 0.187-0.741)$. The remaining factors (VEGF concentration, $\mathrm{HbA}_{1 \mathrm{c}}$ and LDL-C) entered the regression equation with an $\mathrm{OR}>1$, indicating that they were potent risk factors for DPN.

\section{Discussion}

Our study showed that the distribution of 936C/T polymorphism in VEGF 3 '-UTR is prevalent in the population. There were three genotypes: CC, CT and TT. The genotype distribution in the NC group, DM group and DPN group was in accordance with the Hardy-Weinberg equilibrium. Plasma VEGF levels were decreased sequentially in 936CC, CT and TT genotypes. The differences among the 3 genotypes were significant. These data suggest that the 936 $\mathrm{C} / \mathrm{T}$ gene polymorphism in the 3'-UTR of the VEGF gene may lead to reduced VEGF expression. These data are consistent with the results reported by Krippl et al. [7], Han et al. [11] and Morohashi et al. [12]. However, these findings are inconsistent with the results reported by Awata [13]. This inconsistency may be due to the different detection methods and the different sources of VEGF. Kripple [7], Han [11], Morohashi and our lab measured the level of VEGF in the plasma by the ELISA method. The ELISA kits we used could only specifically detect VEGF121 and VEGF165. In contrast, Awata measured the level of VEGF in the serum. During the activation of platelets, large amounts of VEGF were released, which made the serum levels of VEGF often several times higher than the plasma levels of VEGF [13]. This may be an important cause of the inconsistency.

Another finding of this study was that the CC genotype frequency in the DPN group was higher than that in the NC group and DM group, while the frequency of the CT+TT genotype (carrying the T allele) in the DPN group was significantly lower than the DM group and NC group. However, there were no significant differences in the CC, CT and TT genotype distribution between the DM group and NC group. Further analysis found interesting results. Compared with the DM group and NC group, the $C$ allele frequency in the DPN group was higher. The T allele frequency in the DPN group was significantly lower than that in the DM group and NC group, while between the DM group and NC group, there were no significant differences in the $C$ allele frequency and $T$ allele frequency. These data suggest that VEGF gene polymorphism may be associated with DPN pathogenesis but irrelevant for the incidence risk of type 2 diabetes. In diabetic patients, the incidence of DPN in patients with CC genotype was $57.97 \%$, higher than in patients with the CT+TT genotype (carrying the T allele) (39.29\%). The OR value was 2.132, indicating that compared with patients carrying the $\mathrm{CT}+\mathrm{TT}$ genotype, the rela-

Table N. Logistic analysis of multiple risk factors for the association with DPN in type 2 diabetes patients

\begin{tabular}{|lccccccc|}
\hline Variable & \multicolumn{7}{c|}{$\mathrm{Cl}$} \\
\cline { 2 - 7 } & $\beta$ & $\mathrm{SE}$ & $\chi^{2}$ & Value of $p$ & OR & Lower & Upper \\
\hline $\mathrm{HbA}_{1 \mathrm{c}}$ & 0.298 & 0.101 & 8.715 & 0.003 & 1.347 & 1.105 & 1.641 \\
\hline $\mathrm{TC}$ & 0.382 & 0.147 & 6.745 & 0.009 & 1.465 & 1.098 & 1.955 \\
\hline LDL-C & 0.650 & 0.265 & 6.004 & 0.014 & 1.915 & 1.139 & 3.219 \\
\hline VEGF concentration & 0.574 & 0.148 & 15.130 & $<0.001$ & 1.776 & 1.330 & 2.372 \\
\hline VEGF936C $\rightarrow T$ & -1.132 & 0.421 & 6.389 & 0.004 & 0.461 & 0.187 & 0.741 \\
\hline
\end{tabular}


tive occurrence risk of DPN in patients carrying the CC genotype was 2.132 times. These findings suggest that the $936 \mathrm{C} / \mathrm{T}$ polymorphism in the $3^{\prime}-$ UTR of the VEGF gene is closely related to the incidence of DPN. The $C$ allele may be a genetic marker of DPN occurrence, while the T allele may be a protective genetic marker of DPN. These data are consistent with studies reported by TavakkolyBazzaz [14].

The clinical and biochemical features such as blood pressure and glycolipid metabolic disorder between the CC genotype group and the CT+TT genotype group showed no significant difference. However, the levels of blood glucose, blood lipid, $\mathrm{HbA}_{1 \mathrm{c}}$ and VEGF expression of the 936CC genotype group were higher than those of the CT and TT genotype group. Similarly, levels of these clinical and biochemical features in the CT genotype group were also higher than those of the TT genotype group. Another study reported by Chowdhury et al. [15] found that DNA can become glycosylated under a high glucose environment, leading to increased frequency of gene mutations and abnormal gene expression. Therefore the following questions need further study. As we know, the metabolic disorders in genetically susceptible individuals are more difficult to control [16]. It is still not known whether loss of control of metabolic disorder promotes the gene mutation causing the high incidence of DPN. It needs to be further studied in the future whether the abnormal gene expression promotes glucose and lipid metabolic disorders, resulting in high levels of VEGF and increased risk of occurrence of DPN.

The occurrence of DPN is the outcome of the interaction of genetic and environmental factors [17]. To exclude the effect of the interaction and screen out the most meaningful indicators of DPN, we assessed the role of many independent variables such as age, BMI, blood pressure, glycated hemoglobin, plasma C-peptide levels, blood lipids and genotype in the occurrence of DPN by logistic regression analysis. Logistic regression analysis showed that VEGF gene polymorphism was negatively correlated with DPN and that VEGF gene polymorphism may reduce the incidence risk of DPN. The factors $\mathrm{HbA}_{1 c}$, LDL-C and VEGF entered the regression equation with an $O R>1$. So we assumed that they were risk factors for DPN occurrence. These data suggest that glycolipid metabolic disorder plays an important role in the occurrence and development of DPN.

In conclusion, the present study provides an insight into the association of $936 \mathrm{C} / \mathrm{T}$ gene polymorphism in the 3'-UTR of the VEGF gene with DPN. We have shown that the $936 \mathrm{C}$ to $T$ mutation in the 3'-UTR of VEGF may be a protective factor against DPN and negatively correlated with the level of plasma VEGF and the incidence of diabetic peripheral neuropathy. The correlations between gene polymorphisms and glucose and lipid metabolic disorders indicate that the occurrence of DPN is related to the interaction of genetic and environmental factors. Thus the prompt improvement and treatment of high blood glucose, high blood lipid, hypoxia and anemia caused by DM may reduce the risk of occurrence of DPN.

\section{Acknowledgments}

Xinhuan Zhang and Zhongan Sun contributed equally to this work.

This work is supported by the Youth Research Fund of Taishan Medical School (No. 2006ZR029).

\section{References}

1. Ferrara N, Davis-Smyth T. The biology of vascular endothelial growth factor. Endocr Rev 1997; 18: 4-25.

2. Deguchi T, Hashiguchi T, Horinouchi S, et al. Serum VEGF increases in diabetic polyneuropathy, particularly in the neurologically active symptomatic stage. Diabet Med 2009; 26: 247-52.

3. Cameron NE, Cotter MA. Vascular changes in animal models of diabetic neuropathy. J Neurochem 2003; 85 (Suppl. 2): 14.

4. Sondell M, Lundborg G, Kanje M. Vascular endothelial growth factor has neurotrophic activity and stimulates axonal outgrowth, enhancing cell survival and Schwann cell proliferation in the peripheral nervous system. J Neurosci 1999; 19: 5731-40.

5. Tremolada G, Lattanzio R, Mazzolari G, Zerbini G. The therapeutic potential of VEGF inhibition in diabetic micro vascular complications. Am J Cardiovasc Drugs 2007; 7: 393-8.

6. Renner W, Kotschan S, Hoffmann C, et al. A common 936 $\mathrm{C} / \mathrm{T}$ mutation in the gene for vascular endothelial growth factor is associated with vascular endothelial growth factor plasma levels. J Vasc Res 2000; 37: 443-8.

7. Krippl P, Langsenlehner U, Renner W, et al. A common $936 \mathrm{C} / \mathrm{T}$ gene polymorphism of vascular endothelial growth factor is associated with decreased breast cancer risk. Int J Cancer 2003; 106: 468-71.

8. Zhao MD, Cai DH, Chen H. Diabetic neurological complications. Practical endocrinology. Edition 3. Beijing: People's Medical Press 2004; 1414-23.

9. American Diabetes Association American Academy of Neurology. Report and recommendations of the San Antonio Conference on diabetic neuropathy (consensus statement). Dietetic Crae 1988; 11: 592-7.

10. Tang XF. Clinical EMG. Chinese Peking Union Medical College Press, Beijing 1995; 134.

11. Han SW, Kim GW, Seo JS, et al. VEGF gene polymorphisms and susceptibility to rheumatoid arthritis. Rheumatology (Oxford) 2004; 43: 1173-7.

12. Morohashi K, Takada T, Omori K, Suzuki E, Gejyo F. Vascular endothelial growth factor gene polymorphisms in Japanese patients with sarcoidosis. Chest 2003; 123: 1520-6.

13. Awata T, Inoue K, Kurihara S, et al. A common polymorphism in the 5 '-untranslated region of the VEGF gene 
is associated with diabetic retinopathy in type 2 diabetes.

Diabetes 2002; 51: 1635-9.

14. Tavakkoly-Bazzaz J, Amoli MM, Pravica V, et al. VEGF gene polymorphism association with diabetic neuropathy. Mol Biol Rep 2010; 37: 3625-30.

15. Chowdhury TA, Dyer PH, Kumar S, et al. Genetic determinants of diabetic nephropathy. Clin Sci 1999; 96: 221-30.

16. Amer MA, Ghattas MH, Abo-Elmatty DM, et al. Evaluation of glutathione S-transferase P1 genetic variants affecting type-2 diabetes susceptibility and glycemic control. Arch Med Sci 2012; 8: 631-6.

17. Monastiriotis C, Papanas N, Veletza S, et al. APOE gene polymorphisms and diabetic peripheral neuropathy. Arch Med Sci 2012; 8: 583-8. 